\title{
Visual modeling application in the aircraft emergency evacuation simulation based on Virtools
}

\author{
Chaoying Zhang ${ }^{1, \text { a }}$, Hu Liu ${ }^{1, b}$ \\ ${ }^{1}$ Beihang University, China \\ azhchy_1989@126.com, baerodesigner@163.com
}

Keywords: emergency evacuation, visual modeling, Virtools, three dimension simulation

\begin{abstract}
Using interactive virtual simulation methods and combining numerical calculation software, the 3D airline emergency evacuation simulation system is established in Virtools platform according to the received data. The system takes full account of the passengers' individual differences during the evacuation process, setting up different member parameters, which influence the passengers' evacuation behavior. In order to fulfill the different types of evacuation requirements in various simulation, it can change evacuation path algorithm, cabin layout data and evacuees component parameters, to get the corresponding evacuation simulation results. Meanwhile, the system interface can change visual angle to realize human-machine interaction freely and reflect the evacuation of the evacuation process accurately. Also the reliability of the system simulation result is verified by compared with the results of actual certification trial.
\end{abstract}

\section{Introduction}

The traditional way of aircraft emergency evacuation test is mainly realized by amount of trials in actual environment, combining with the continuously improved previous experience. This way can't present the whole process directly. Meanwhile the whole process cycle is long, the cost is high, the emergency evacuation process optimization effect is not obvious, and it often needs to pay a big price to exchange for small improvement. With the development of virtual simulation technology, the visual modeling by software platform begins to be applied emergency evacuation training field. The technology can realize the reality of complicated scene in economical means, letting the users experience the feeling of being personally on the scene. With the advantages of low cost, low risk and high safety, the superiority of virtual simulation method gradually emerges in aircraft emergency evacuation test. Therefore, many countries in the world are engaged in developing strong pertinence virtual training simulation software to apply in aerospace field[1,2].

In view of the validity of emergency evacuation simulation, it is necessary to give full consideration the individual differences of participates in evacuation. The civil aircraft emergency evacuation 3D simulation system in this paper uses the interactive virtual simulation method, and combines numerical calculation software, according to the received data[3], to emergency evacuation test on the 3D simulation. The process fully considers the passengers' individual differences, and sets up the corresponding physical parameters according to the different characteristics of members in the process of evacuation, to influence behavior. And in the evacuation interface the angle of view can be freely switched, in order to accurately reflect the evacuation process.

\section{Method of Visualization}

This paper use the developed platform Virtools software, which can be agile manufacture immersive virtual environment to form a variety of sensory experience, giving the participants the feeling of being personally on the scene. The software can be not only used to produce all kinds of 3D games, but also applied to multiple simulation fields[4].

Modeling by Virtools platform must enter a graphical user interface first and write the scripting language of interaction behavior element by the modular behavior module, and then lead into the model created by other modeling tools through the plugin file. At present, in the field of virtual 
simulation modeling a variety of modeling tools are widely used. Considering the quality degree of models, the system resources occupation of modeling tools and the application of comprehensive scale, this paper chose two kinds of modeling tools, Catia and 3DMax. Considering the Catia software fine ability to create complex model, it will be more realistic to present cabin interior scene arrangement of the aircraft fuselage static modeling. At the same time, 3DMax has advantage of creating relatively simple, of which the quality degree requirements is relatively low and the mode is set as dynamic model (such as the crew, evacuation passengers, escape slide, etc.), in order to reduce the system resources consumption and improve the operation speed.

\section{System Realization}

System Framework. The civil aircraft emergency evacuation 3D simulation system set by Virtools totally contains two data files, two function modules and three resource compositions. The system will operate by reading room initial configuration files, aircraft and personnel models and map data. And combined with the actual situation, the initial default seat is set as random according to the number. Then by reading emergency evacuation path data, the whole evacuation process real-time simulation can be realized in the main interface, including display the real-time personnel evacuation position and action, evacuated number, evacuation time display, etc. In the process of simulation switching multiple angle and controlling broadcast schedule can also be realized.

The general framework can be divided into two parts according to the operation procedure and function relationship, specific as follows figure 1.

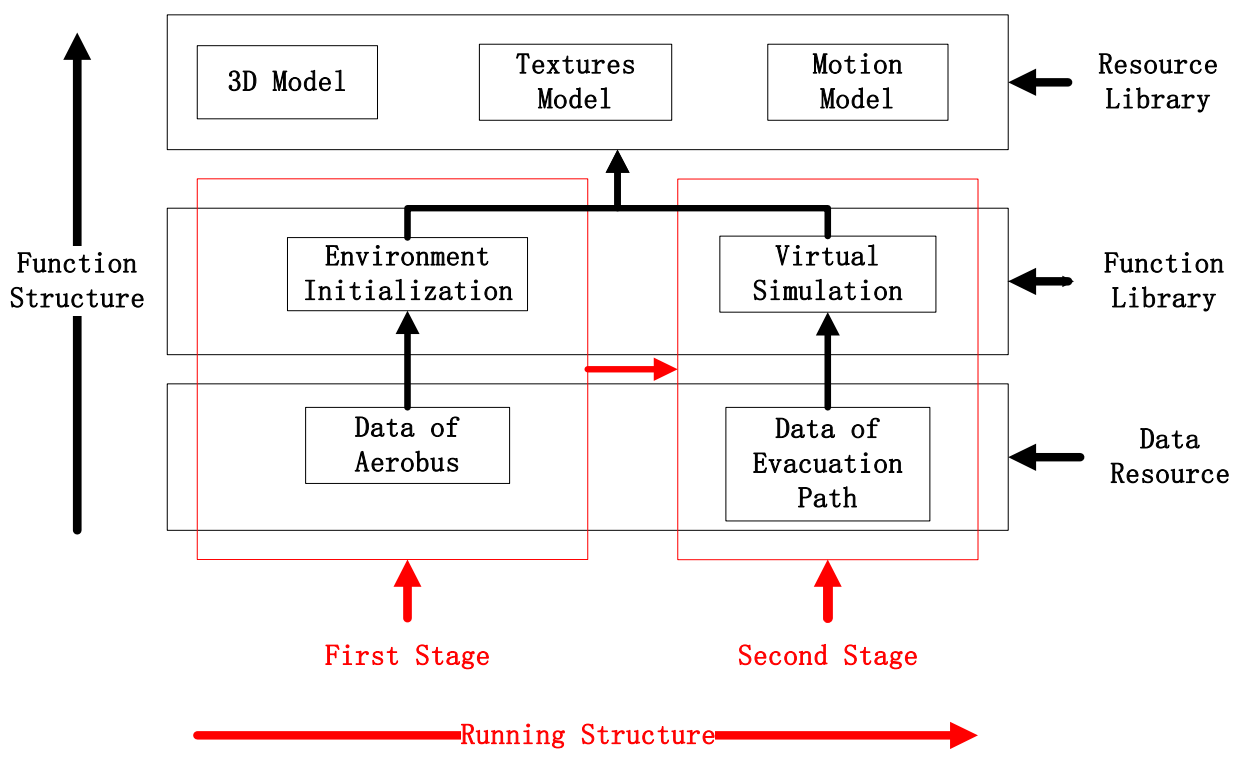

Fig. 1 The overall structure of virtual system

System Constitution. At the beginning of operation, the system will load two data files, respectively engine initial configuration file and personnel emergency evacuation path file. The engine room initial configuration file including cabin basic layout information, including the cockpit, fuselage, wing, passenger seat, toilet, toilet, storage room, kitchen, and emergency exit, position and size; The cabin all kinds of obstacles (such as blanket, cushion for leaning on, etc.) distribution; The passengers and aircraft crew group composition, quantity and the initial position. And personnel emergency evacuation path data file contains the number of passenger evacuation and each passengers real-time path information, including time point, the position coordinates, target export. The loaded simulation interface is shown in figure2.

The system also includes two function modules, the simulation environment initialization and real-time simulation. The former can be set according to the engine room initial configuration files, corresponding planes, characters, such as object model and map loading, complete the initial simulation environment to create; Camera angle default initialized to order perspective; All 
personnel default initialization action to "sit". And real-time simulation module according to personnel emergency evacuation path data file, the simulation of the simulation process. The concrete content includes reading file evacuees quantity, and the corresponding displayed in the interface state hint bar "evacuation total number" behind. Read the file each passenger, air crew, crew position coordinates information, according to its identity (passengers, air crew, crew), location, reaction time and so on to determine its current motion and dynamic display. Evacuation process, the software provides a ten observation angle, including eight fixed angle of view, a top view and a around perspective, can be easily switched according to demand, and carries on the comprehensive process observation (sample in figure 3). Every successful evacuation one person, interface above the status bar "have been evacuated number" will be dynamic increase one, until all the evacuation. The whole evacuation process will be in the interface when the status bar of the "evacuation time" display.

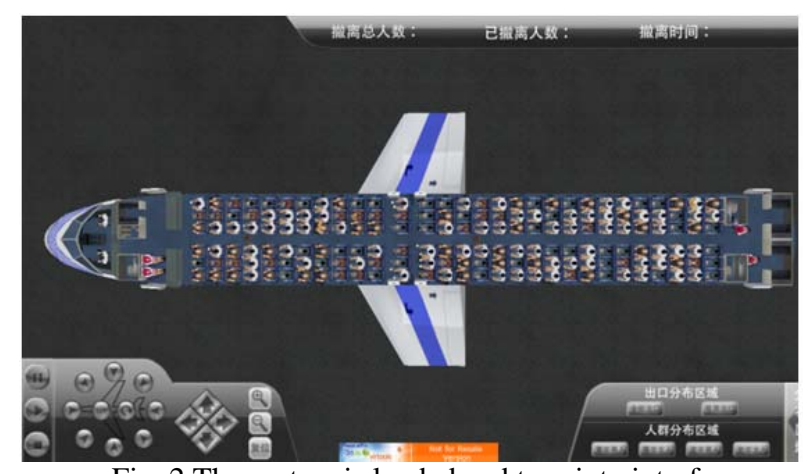

Fig. 2 The system is loaded and turn into interface

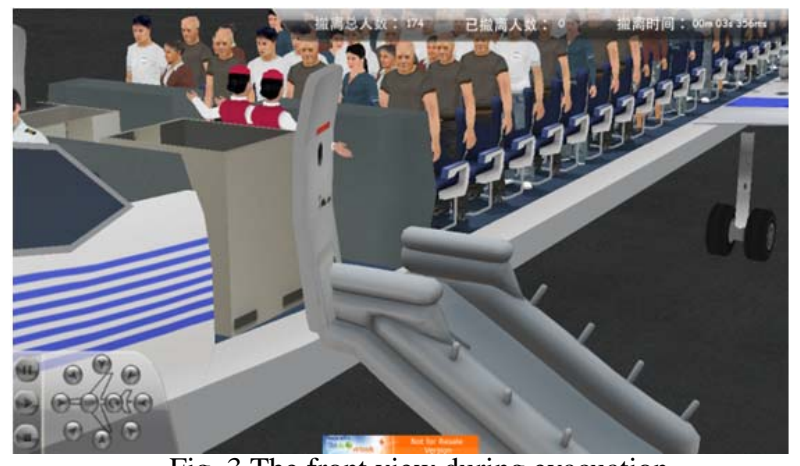

Fig. 3 The front view during evacuation

In order to maximize the reality of simulation, the system designs the rich data repository, mainly including 3D model database, mapping material resource repository and action database. Among them, the 3D model database including aircraft, passengers, air crew, obstacles and model. And mapping material resource is the model of the map and material file. Action repository is including the cabin all personnel action (sit, up, walk, jump, etc.) and the body action (open the door, put down the escape slide).

Operation Process. When click start command, the crew rise and make evacuation gestures. At the same time, the escape doors open, and the escape slides are set down. Meanwhile, all the passengers stand up and are ready to leave according to the loaded path. Then all the passengers begin to leave according to their evacuation route calculated by path selection algorithm. While all the other passengers evacuated after completion, the air crew begin to leave. After all the people leaving the aircraft, the system will export the evacuation report.

\section{Sample Test and Validation}

To validate the reliability of the system, the paper refers the research result of Gelea[5,6]. The system carries out three tests on different condition, and compares the results with those given by Gelea carried out by actual certification trial in the similar conditions. The initial conditions of the three contrast experiment are set as below.

Test 1: Evacuation number of passengers is 159 , only the single side three doors in the aircraft can be open;

Test 2: Evacuation number of passengers is 180, only the single side three doors in the aircraft can be open;

Test 3: Evacuation number of passengers is 159, all the six doors in the aircraft can be open.

The average evacuation time of the three contrast experiments are shown in the following table 1. 
Table 1 the result of three different tests

\begin{tabular}{ccc}
\hline Test & Simulation System & $\begin{array}{c}\text { Actual } \\
\text { CertificationTrial }\end{array}$ \\
\hline Test 1 & 76.4 & $70.5 \mathrm{~s}$ \\
Test 2 & $81 \mathrm{~s}$ & $73 \mathrm{~s}$ \\
Test 3 & $67 \mathrm{~s}$ & $62.1 \mathrm{~s}$ \\
\hline
\end{tabular}

Through the comparison, the average evacuation time of the simulation system is longer than the actual certification trial, but it is in the permitted rang in general. The reason of that can be the simulation considers the congestion and wait during evacuation, which may be caused by panic during real escape. Thus, it is can be concluded that the civil aircraft emergency evacuation 3D simulation system can simulate the real evacuation trial and also the result of the simulation is reliable to some extent. Also, it can be used in some new civil aircraft evacuation test someday in the future.

\section{References}

[1] C. Burstedde, K. Klauck, A. Schadschneider, et al. Simulation of pedestrian dynamics sing a two-dimensional cellular automaton[J]. Physica A, 295 (2001): 507-525.

[2] Sharad Sharma, Harpreet Sinch, Atul Prakash. Multi-Agent Modeling and Simulation of Human Behavior in Aircraft Evacuations. Transactions on aerospace and electronic systems, 2008, vol.44:1477-1488.

[3] Zhang Yu-gang, Song Bi-feng, Xue Hong-jun, et al. Simulation Analysis of CivilAircraft Emergency Evacuation Impact Factor. Computer Simulation. 28(6):63-74(2011).

[4 ]Geng Wentao, Wu Xudong, Liu Hu, Wu Zhe. Simulation of Missile Loaded to Fighter Based on Virtools. Aircraft Design. 29(1):51-56 (2009).

[5] Galea E R, Blake S J, Gwynne S A. Methodology and procedure for the introducationof aircraft evacuation simulation to the aircraft certification process[R]. VERRES Project, GMA2/200/32039, 2003.

[6] Galea E R, Blake S J, Lawrence P J. Report on the Testing and Systematic Evaluation of the airEXODUS Aircraft Evacuation Model. Available in:

http://www.caa.co.uk/docs/33/caapaper2004_05.pdf. 